\title{
Influência da retroalimentação auditiva nos parâmetros acústicos vocais de indivíduos sem queixas vocais
}

\author{
Influence of auditory feedback in the vocal acoustic parameters \\ of individuals without vocal complaints
}

\author{
Luciene da Cruz Fernandes', Dandara Aiodelê Silva Bomfim¹, Gabriela Carvalho Machado², Caio Leônidas de \\ Andrade $^{2}$
}

\section{RESUMO}

\begin{abstract}
Introdução: A qualidade da emissão vocal é dependente da integridade do mecanismo de retroalimentação auditiva. A presença de eventuais falhas nesse mecanismo está relacionada à indução do abuso e/ ou mau uso vocal e, por conseguinte, do surgimento de quadros de disfonia. Objetivo: Avaliar a influência da retroalimentação auditiva na intensidade e na frequência da voz, em indivíduos sem queixas vocais. Métodos: Participaram da pesquisa 40 sujeitos do gênero feminino, sem queixas vocais e com limiares auditivos dentro dos padrões de normalidade. As participantes foram submetidas a uma avaliação auditiva, composta por audiometria tonal liminar, imitanciometria e por uma avaliação acústica vocal dos parâmetros de intensidade e frequência, realizada em três momentos: antes, durante e após a exposição ao ruído branco. Resultados: Houve diferença significativa na relação das médias obtidas na intensidade e entre o limiar dos reflexos acústicos contralaterais e as frequências vocais, nos três momentos da avaliação acústica. Conclusão: Os achados sugerem que a retroalimentação auditiva interfere no controle da intensidade e frequência vocal.
\end{abstract}

Palavras-chave: Retroalimentação; Percepção auditiva; Qualidade da voz; Nível de discriminação sonora; Percepção sonora

\begin{abstract}
Introduction: The quality of the vocal emission is dependent on the integrity of the auditory feedback mechanism of the presence of eventual failures is related to the induction of abuse and / or vocal misuse, and therefore of the surgeon of dysphonia. Purpose: To evaluate the influence of auditory feedback in voice intensity and frequency in individuals with no vocal complaints. Methods: Participants were 40 female subjects without vocal and auditory thresholds within normal standards complaints. Participants underwent an auditory evaluation composed of pure tone audiometry, tympanometry and a vocal acoustic assessment of intensity and frequency parameters, carried out in three stages: before, during and after exposure to white noise. Results: There was significant difference in the average of the ratio obtained in intensity and between the threshold of contralateral acoustic reflexes and vocal frequencies in the three moments of acoustic evaluation. Conclusion: Thus, the findings suggest that auditory feedback interferes with the control of the intensity and vocal frequency.
\end{abstract}

Keywords: Feedback; Auditory perception; Voice quality; Pitch discrimination; Loudness perception

Trabalho realizado no Curso de Fonoaudiologia, Departamento de Ciências da Vida, Universidade do Estado da Bahia - UNEB - Salvador (BA), Brasil.

(1) Curso de Fonoaudiologia, Departamento de Ciências da Vida, Universidade do Estado da Bahia - UNEB - Salvador (BA) Brasil.

(2) Programa de Pós-graduação em Processos Interativos dos Órgãos e Sistemas, Instituto de Ciências da Saúde, Universidade Federal da Bahia - UFBA - Salvador (BA), Brasil.

Conflitos de interesses: Não

Contribuição dos autores: $L C F$ foi responsável pela elaboração da ideia inicial da pesquisa, realizou a submissão do projeto do estudo à Plataforma Brasil, para análise do Comitê de Ética em Pesquisa com seres Humanos, participou da escrita do texto e da análise dos dados; DASB coletou os dados e contribuiu com grande parte da escrita do texto; $C L O A$ e $G C M$ participaram da escrita do texto, realizaram a normatização e as correções finais do artigo.

Autor correspondente: Luciene da Cruz Fernandes. E-mail: luenefernandes@ gmail.com

Recebido: 19/12/2016; Aceito: 21/11/2017 


\section{INTRODUÇÃO}

O principal e fundamental meio de comunicação do ser humano é a voz. A qualidade vocal (QV) permite expressar informações sobre as características físicas e psicossociais, implicando singularidade, personalidade e humor ao falante, configurando-se como um conjunto de características que identificam uma voz ${ }^{(1)}$.

Para o adequado controle da qualidade da emissão vocal, a integridade do sistema auditivo é de primordial importância ${ }^{(1)}$. Mais especificamente, o reflexo acústico estapediano é um mecanismo que tem participação relevante na modulação da intensidade vocal, ou no início de uma vocalização ${ }^{(2,3)}$. Outra função importante desse reflexo é a sua atuação na melhora da discriminação de fala, sobretudo nas altas intensidades e na seletividade das frequências, além de proporcionar melhora da atenção auditiva para sons contínuos e seletividade do sinal auditivo, em detrimento do ruído de fundo ${ }^{(4)}$, o que sugere que o reflexo acústico tem funções importantes na retroalimentação auditiva, em ambientes com ou sem ruído.

$\mathrm{Na}$ presença de eventuais falhas no mecanismo de retroalimentação auditiva, é muito comum observar ausência ou insuficiência de regulação do parâmetro acústico de intensidade vocal, induzindo ao abuso e/ou mau uso vocal e, por conseguinte, ao surgimento dos quadros de disfonia ${ }^{(5,6)}$, situação compatível com uma das etiologias dos casos de rouquidão nos indivíduos, bem como das recidivas frequentes desses sintomas, pós-tratamento.

As mudanças nos parâmetros da voz observadas durante o discurso diferem daquelas que ocorrem durante o ato voluntário de 'falar alto', como, por exemplo, quando um locutor simplesmente pede para falar mais forte, ou quando um professor eleva a intensidade da voz para se fazer entender por estudantes em uma grande sala de aula. Tal constatação enfatiza o efeito Lombard, que se caracteriza pelo aumento da amplitude vocal em resposta a um aumento no ruído de fundo, como um reflexo natural, e pode ser uma indicação de que diferentes mecanismos de controle neural estão envolvidos na mudança voluntária e involuntária da amplitude da $\operatorname{voz}^{(7)}$.

Para que se tenha uma boa QV, é necessária a ação eficaz do sistema de retroalimentação auditiva. Conhecer a sua funcionalidade torna-se fundamental para a prevenção de patologias vocais, principalmente em indivíduos que fazem uso da voz profissionalmente e que estão expostos, diariamente, a ambientes ruidosos. Assim, este estudo teve como objetivo investigar de que modo a retroalimentação auditiva pode influenciar nos parâmetros vocais acústicos de indivíduos sem queixas vocais e sem alterações nos limiares auditivos tonais.

\section{MÉTODOS}

Trata-se de um estudo descritivo e analítico, realizado em uma clínica-escola de fonoaudiologia, em Salvador, Bahia, durante o período de maio a junho de 2014, aprovado pelo Comitê de Ética e Pesquisa da Universidade do Estado da Bahia (UNEB), sob o protocolo número 467.932. Todos os sujeitos que concordaram em participar da pesquisa assinaram o Termo de Consentimento Livre e Esclarecido.

A amostra do estudo foi obtida por conveniência, composta por 40 indivíduos do gênero feminino, uma vez que na literatura há mais relatos de estudos envolvendo mulheres ${ }^{(8,9)} \mathrm{e}$ devido a maior facilidade de voluntariado. Todas eram adultas, sem queixas vocais e apresentaram limiares auditivos dentro dos padrões de normalidade.

Inicialmente, foi aplicado o questionário de avaliação da $\mathrm{voz}^{(1)}$, para investigar os fatores de risco aos quais as participantes estavam expostas, bem como a sua rotina e as condições gerais de saúde.

Posteriormente, foram realizadas as avaliações auditivas, com os procedimentos de meatoscopia, audiometria tonal liminar nas frequências de $250 \mathrm{~Hz}$ a $8000 \mathrm{~Hz}^{(10)}$, bem como a pesquisa do limiar de reconhecimento da fala (LRF) e pesquisa do índice de reconhecimento da fala (IPRF), utilizando o audiômetro digital Interacoustics ${ }^{\circledR}$ AC-30. Medidas de imitância acústica foram realizadas por meio do analisador de orelha média (imitanciômetro) Interacoustics ${ }^{\circledR}$ AZ 7, a fim de verificar a mobilidade tímpano-ossicular e os reflexos acústicos contralaterais, nas frequências de $500 \mathrm{~Hz}, 1000 \mathrm{~Hz}, 2000 \mathrm{~Hz}$ e $4000 \mathrm{~Hz}$.

As participantes incluídas no estudo passaram por uma avaliação espectrográfica da voz, quando foi solicitado que emitissem a vogal /a/ sustentada, fala sequencial (dias da semana) e fala encadeada (cantar o "parabéns")(11). Para esta avaliação, os equipamentos utilizados foram: notebook emachines D442-V081, fone de ouvido com microfone clone e software PRAAT- analisador vocal.

As amostras foram gravadas em programa específico para análise vocal, o software PRAAT, e, em seguida, submetidas à análise espectrográfica, para a obtenção das medidas de frequência (pitch) e intensidade (loudness). Levou-se em consideração o valor médio do pitch e do loudness de cada amostra vocal, fornecidos nos ícones "voice reports" e "intensity", respectivamente, ambos localizados na janela de análise do PRAAT.

As vozes das participantes foram gravadas em três momentos: antes, durante e após a exposição ao ruído. No segundo momento, elas foram expostas a ruído branco, de $80 \mathrm{~dB}$ NPS de intensidade e duração de 100 segundos, através dos fones de ouvido inseridos em ambas as orelhas.

As gravações foram comparadas de acordo com o seguinte agrupamento: média do momento antes da exposição e média do momento durante a exposição; média do momento antes da exposição com média do momento pós-exposição. Foi comparada, também, a relação dos momentos antes e após a exposição com os reflexos acústicos contralaterais avaliados. Ou seja, para a análise de cada agrupamento, obteve-se a média do valor médio do pitch e do loudness de cada amostra vocal. 
Para as análises, utilizou-se o programa estatístico SPSS, versão 17.0. Foi realizado o teste t pareado e a correlação de Pearson, considerando-se o nível de significância de 5\% (p $\leq$ $0,05)$.

\section{RESULTADOS}

A amostra foi composta por 40 participantes do gênero feminino, com média de idade de 26,6 $( \pm 8,5)$ anos. Foram calculadas as médias das frequências e das intensidades vocais dos três momentos da gravação. Observou-se diferença entre as médias das intensidades nos períodos antes e durante a exposição ao ruído e antes e após a exposição (Tabela 1).

Ao comparar as respostas do reflexo acústico com a emissão vocal antes e após a exposição ao ruído, nos aspectos de intensidade e frequência, (Tabela 2), observou-se ausência de correlação entre os reflexos acústicos e diferença entre os momentos antes e após a exposição ao ruído, no aspecto intensidade. Houve diferença entre o limiar dos reflexos acústicos e os distintos períodos antes e depois da exposição ao ruído, no aspecto da frequência, sendo em $1000 \mathrm{~Hz}(\mathrm{p}=0,05$ e r=0,53) e $2000 \mathrm{~Hz}(\mathrm{p}=0,01$ e $\mathrm{r}=0,85)$.

\section{DISCUSSÃO}

De acordo com a análise dos resultados obtidos na pesquisa, observou-se diferença na relação das médias obtidas na intensidade entre os momentos antes e durante a exposição ao ruído e os momentos antes e após a exposição ao ruído das amostras vocais. Também foi possível observar diferença entre a correlação dos reflexos acústicos e as amostras vocais dos momentos antes e depois da exposição ao ruído.

Constatou-se média de intensidade vocal em condições habituais de 65,6 $( \pm 6,2) \mathrm{dB}$, assemelhando-se aos resultados de uma investigação com 58 indivíduos adultos, em que a média do momento sem exposição ao ruído foi de $63,8 \mathrm{~dB}$, para o gênero feminino ${ }^{(12)}$.

Na condição de exposição ao ruído, foi possível observar o aumento da média de intensidade para $70,5( \pm 6,2) \mathrm{dB}$, tornando a amostra estatisticamente significativa, quando comparada à média de intensidade obtida antes da exposição ao ruído. Achados na literatura especializada demonstraram o aumento significativo da intensidade, com o aumento dos níveis de ruído de mascaramento, confirmando a teoria do efeito Lombard, em que a intensidade vocal aumenta sistematicamente com o aumento do nível de ruído ${ }^{(13)}$.

Após a exposição ao ruído, a média da intensidade diminuiu, em relação ao período durante a exposição ao ruído, porém, manteve-se maior que o valor antes da exposição. Este fato está relacionado a um processo normal, ocasionado pela diminuição da percepção sonora, uma vez que, para que haja a estabilização vocal, é necessário um tempo, que pode variar de indivíduo para indivíduo. Este resultado já foi observado na literatura, que afirmou que a ausência de monitoramento auditivo gera mudanças, caracterizadas, principalmente, por descontrole na intensidade e deslocamento da frequência fundamental ${ }^{(14)}$.

Tabela 1. Comparação entre os valores médios e desvios-padrão, nos três diferentes momentos das análises vocais

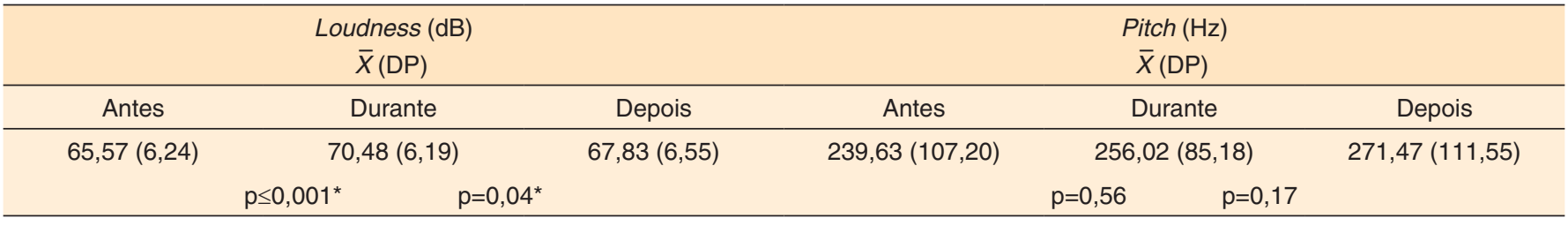

${ }^{*}$ Valores significativos $(p \leq 0,05)$ - teste T pareado

Legenda: DP = desvio padrão

Tabela 2. Distribuição dos resultados obtidos através da correlação entre os reflexos acústicos e a diferença entre o momento antes e após da gravação vocal

\begin{tabular}{|c|c|c|c|c|c|c|c|c|c|c|}
\hline \multirow{2}{*}{\multicolumn{3}{|c|}{ Reflexos acústicos (dB) }} & \multicolumn{4}{|c|}{$\begin{array}{l}\text { Correlação dos reflexos acústicos com o } \\
\text { diferencial loudness antes/depois } \\
\qquad(\bar{X}=1,80 \pm 3,28 \mathrm{~dB})\end{array}$} & \multicolumn{4}{|c|}{$\begin{array}{l}\text { Correlação dos reflexos acústicos com o } \\
\text { diferencial pitch antes/depois } \\
(\bar{X}=45,87 \pm 117,00 \mathrm{~Hz})\end{array}$} \\
\hline & & & \multicolumn{2}{|c|}{ Orelha direita } & \multicolumn{2}{|c|}{ Orelha esquerda } & \multicolumn{2}{|c|}{ Orelha direita } & \multicolumn{2}{|c|}{ Orelha esquerda } \\
\hline $\begin{array}{c}\text { Frequências } \\
(\mathrm{Hz})\end{array}$ & $\begin{array}{l}\text { Orelha } \\
\text { direita }\end{array}$ & $\begin{array}{c}\text { Orelha } \\
\text { esquerda }\end{array}$ & $p$ & $r$ & $p$ & $r$ & $\mathrm{p}$ & $r$ & $\mathrm{p}$ & $r$ \\
\hline 500 & $98,42 \pm 8,34$ & $99,74 \pm 7,54$ & 0,39 & 0,22 & 0,22 & $-0,32$ & 0,48 & 0,21 & 0,09 & $-0,49$ \\
\hline 1000 & $96,58 \pm 8,98$ & $95,28 \pm 8,13$ & 0,31 & 0,27 & 0,35 & $-0,29$ & $0,05^{\star}$ & 0,53 & 0,35 & $-0,30$ \\
\hline 2000 & $92,37 \pm 8,72$ & $93,16 \pm 8,20$ & 0,17 & 0,36 & 0,48 & $-0,19$ & $\leq 0,01^{*}$ & 0,85 & 0,39 & $-0,26$ \\
\hline 4000 & $94,71 \pm 8,74$ & $93,68 \pm 5,97$ & 0,96 & $-0,01$ & 0,28 & $-0,28$ & 0,12 & 0,50 & 0,61 & $-0,15$ \\
\hline
\end{tabular}

*Valores significativos $(p \leq 0,05)$ - teste de Correlação de Pearson

Legenda: $p=$ nível de significância; $r$ = nível de correlação 
Outros pesquisadores ${ }^{(15)}$ observaram que o sistema de controle auditivo vocal pode ser influenciado por causas inatas ou diferenças individuais, sendo as frequências e intensidades controladas de forma independente em nível neuronal. Há também a hipótese de que o aumento da intensidade sob ruído mascarante seja gerado por uma variedade de estratégias que podem se diferenciar fisiologicamente entre as pessoas ${ }^{(13)}$.

Quanto aos aspectos da frequência, a média encontrada, na condição inicial, foi de $239,6 \mathrm{~Hz}( \pm 107,2)$. Ao ser introduzido o ruído, obteve-se a média de $256,0 \mathrm{~Hz}( \pm 85,2)$. Esta elevação já era estimada, devido à condição de exposição. Após cessar o ruído, a média da frequência foi de $271,5 \mathrm{~Hz}( \pm 111,6)$. Neste estudo não se observou diferença entre as médias das frequências obtidas, quando comparados os momentos antes e durante a exposição ao ruído e os momentos antes e após a exposição.

Estes resultados divergem de um estudo sobre a análise das modificações vocais em repórteres na situação de ruído, realizado com 46 sujeitos, sendo 23 repórteres (grupo caso) e 23 não repórteres (grupo controle), em que os valores acústicos de variabilidade de frequência em $\mathrm{Hz}$ foram maiores na presença de ruído mascarante, pois, o aumento da intensidade vocal gerou o deslocamento da frequência fundamental, já que ambos os grupos apresentaram aumento das médias de intensidade e frequência, quando foram inseridos $50 \mathrm{~dB}$ e 90 $\mathrm{dB}$ de mascaramento ${ }^{(16)}$.

A duração da exposição ao ruído utilizada para este estudo foi de, aproximadamente, dois minutos e, neste período, já foi possível observar as modificações dos aspectos vocais de intensidade e frequência. Se comparada ao tempo de exposição ao ruído de um trabalhador que utiliza a voz como seu instrumento principal de trabalho, cuja carga horária mínima é de seis a oito horas, pode-se sugerir que essas modificações fisiológicas de frequência e intensidade se dariam em um grau ainda mais elevado, levando-o a acometimentos mais intensos.

Em uma investigação sobre incidência de disfonia em professores, verificou-se que, após a utilização da voz por horas seguidas, podem ocorrer modificações vocais, caracterizadas, acusticamente, pelo espectro com diminuição de energia na proporção harmônicos/ruído e modificações na frequência fundamental, assim como a exposição ao ruído durante várias horas contínuas provoca prejuízos no processo de retroalimentação auditiva, levando o indivíduo a uma dificuldade maior em controlar os aspectos vocais, após cessar o ruído ${ }^{(17)}$.

Observou-se, neste estudo, que houve diferença estatisticamente significativa quando avaliado o aspecto vocal de intensidade isoladamente, apenas comparando as médias entre si. Já quando correlacionada a intensidade vocal com os reflexos acústicos, não houve diferença. Ao contrário, no aspecto frequência, que ao ser avaliado isoladamente, apesar da frequência fundamental ser maior após o ruído mascarante, não se verificou diferença, porém, quando correlacionada com os reflexos acústicos, foi possível observá-la.
Pode-se sugerir, contudo, que, quando correlacionados os reflexos acústicos com as amostras vocais, no aspecto de intensidade, justifica-se a diferença pela participação desses reflexos na modulação da intensidade vocal e na seletividade das frequências de fala.

Nas mulheres, as pregas vocais vibram aproximadamente 250 vezes por segundo para gerar um enunciado. Quando o volume da voz é elevado de forma sistemática, gera-se um sobre-esforço fonatório, que pode provocar alteração vocal. A literatura $^{(18)}$ descreveu que mulheres estão mais predispostas a desenvolverem fadiga vocal, uma vez que a voz feminina possui frequência fundamental maior, quando comparada com a voz masculina.

A retroalimentação auditiva é um sistema que monitora auditivamente a fala sequencial e, para que haja o monitoramento efetivo, é necessária a garantia da integridade da via auditiva eferente, sendo a resposta reflexa estapediana aos sons de forte intensidade sonora uma das mais evidentes, constantes e estáveis, dentre as respostas eferentes da via auditiva ${ }^{(19)}$. Para além desta função, o reflexo acústico também é responsável pela melhora na discriminação de fala em fortes intensidades e na seletividade de frequência, justificando, assim, a relação entre o reflexo acústico e a diferença de frequência, antes e após o ruído mascarante ${ }^{(20)}$.

Neste estudo, foi possível observar que os limiares dos reflexos acústicos encontrados nas frequências de $1000 \mathrm{~Hz}$ e $2000 \mathrm{~Hz}$ tiveram correlação positiva com os limiares antes e depois da exposição ao ruído, ou seja, quanto maior o limiar do reflexo acústico, maior a diferença entre o momento antes e o momento depois da exposição ao ruído.

Outra observação interessante do presente estudo diz respeito à predileção da lateralidade, na qual se verificou que as respostas reflexas obtidas revelaram significância apenas na orelha direita, em $50 \%$ das frequências avaliadas. Estes achados concordam com a teoria de que o sistema auditivo central e periférico funciona de forma lateralizada, em que a atividade coclear à direita é mais significativa que à esquerda, porém com predominância irregular em toda extensão coclear ${ }^{(21)}$. Achados clínicos apontaram o trato olivococlear medial (TOM) como componente fundamental na manutenção do padrão da assimetria periférica, sendo a modulação da função coclear mediada pelo córtex, através das fibras eferentes ${ }^{(22)}$.

Evidências de melhores respostas dos potenciais evocados auditivos do tronco encefálico, com maiores amplitudes da onda III, bem como nos registros dos sinais das emissões otoacústicas na orelha direita, em contrapartida com a maior prevalência de queixas de zumbido e diagnóstico de perda auditiva na orelha esquerda, confirmam a teoria da assimetria entre as orelhas e sugerem que a orelha esquerda é mais susceptível a alterações auditivas, especialmente quando exposta a ruído ocupacional $^{(23,24,25,26)}$.

Os neurônios dopaminérgicos do TOM são quase que exclusivamente observados na porção basal ou medial da cóclea. 
Nesta região, há indicativos de maior modulação seletiva de frequência, especialmente na faixa de $1-4 \mathrm{kHz}$, na qual se verifica maior efeito de supressão de estimulação acústica contralateral $^{(27)}$.

Pode-se sugerir, portanto, que há maior probabilidade da ocorrência de falha na retroalimentação auditiva para os sons de média frequência, o que resultou no aumento significativo da frequência vocal. Neste sentido, classifica-se a retroalimentação auditiva frequência-alterada como a variação da frequência da voz do falante, usualmente entre $1 / 4$ a 1/8, acima ou abaixo. Com este recurso, o falante ouve a própria voz em uma frequência diferente da usual ${ }^{(28)}$.

A descarga aferente dos receptores sensoriais em resposta a alterações na laringe e condições vocais do trato é transmitida para o tronco cerebral e, em seguida, para os neurônios motores dos músculos da laringe. Este sistema do reflexo intrínseco da laringe pode ser particularmente ativado quando a retroalimentação auditiva é falha ou interrompida ${ }^{(13)}$.

Por isso, na população com falha no mecanismo de retroalimentação auditiva há aumento da frequência fundamental média, em decorrência da ausência de retroalimentação acústica, quando é comum que produza mais esforço vocal para se fazer entender, durante a comunicação oral ${ }^{(29)}$, o que justifica os achados desta pesquisa. Em contrapartida, em um estudo sobre a correlação entre o ruído ambiente em sala de aula e a voz do professor, não foi confirmada a hipótese levantada em muitas publicações, de que a presença de ruído ambiental estaria relacionada à presença de vozes alteradas, entre professores ${ }^{(30)}$.

Diante das análises realizadas, pode-se inferir que os achados deste estudo são compatíveis com os achados da literatura, podendo contribuir com o desenvolvimento de estudos de cunho preventivo, no âmbito da higiene vocal, bem como no estudo do tratamento para qualidade vocal $(\mathrm{QV})$ de indivíduos que fazem uso da voz profissionalmente e que estão expostos, diariamente, a ambientes ruidosos, bem como daqueles com deficiência auditiva.

Ainda se faz necessária, entretanto, a continuidade da definição de valores normativos para diferentes faixas etárias e gêneros, para adequar a prática clínica, tal como melhor entendimento dessa relação tão complexa entre a voz e a audição.

\section{CONCLUSÃO}

A condição de exposição ao ruído mascarante provocou aumento da intensidade da voz dos trabalhadores e a interrupção da exposição ao ruído ocasionou diminuição da intensidade vocal. Houve correlação positiva entre os reflexos acústicos e a diferença de frequência entre os momentos antes e após a exposição ao ruído mascarante.

Desta forma, a retroalimentação auditiva é fundamental no controle dos parâmetros vocais e o seu treinamento em pessoas com alterações vocais pode-se fazer necessário para a eficácia do tratamento e prevenção das patologias vocais.

\section{REFERÊNCIAS}

1. Behlau M, Pontes P. Voz: o livro do especialista. Rio de Janeiro: Revinter; 2004. Vol 1, p. 348.

2. Simmons FB. Perceptual theories of middle ear muscle function. Trans Am Otol Soc. 1964;52:114-31.

3. Borg E, Zakrisson JE. Stapedius reflex and monoaural masking. Acta Otolaryngol. 1974;78(3-4):155-61.

4. Wodmald PJ, Rogers C, Gatehouse S. Speech discrimination in patients with Bells palsy and a paralysed stapedius muscle. Clin Otolaryngol. 1995;20(1):59-62. https://doi. org/10.1111/j.1365-2273.1995.tb00013.x

5. Ishii C, Arashiro PM, Pereira LD. Ordenação e resolução temporal em cantores profissionais e amadores afinados e desafinados. Pro Fono. 2006;18(3):285-92. https://doi.org/10.1590/S010456872006000300008

6. Guimarães RV. Breve revisão sobre a disfemia. Profala. [s.d.] [Acesso em: 28 maio 2013]. Disponível em: http://www.profala.com/ arttf40.htm

7. Buosi MMB, Ferreira LP, Momensohn-Santos TM. Percepção auditiva de professores disfônicos. Audiol Commun Res. 2013;18(2):101-8. https://doi.org/10.1590/S2317-64312013000200008

8. Brum DM. Modificações vocais e laríngeas ocasionadas pelo som basal. Santa Maria [Dissertação]. Santa Maria: Universidade Federal de Santa Maria; 2006.

9. Schwarz K. Modificações laríngeas e vocais produzidas pelo som vibrante lingual. Santa Maria [Dissertação]. Santa Maria: Universidade Federal de Santa Maria; 2006.

10. Zollinger SA, Brumm H. The Lombard effect. Curr Biol. 2011;21(16):614-5. https://doi.org/10.1016/j.cub.2011.06.003

11. Pinho SR, Pontes P. Escala de avaliação perceptiva da fonte glótica: RASAT. 2002 [Acesso em: 10 out 2013]. Disponível em: http://www. invoz.com.br/paginas/rasat_pt.pdf

12. Koishi HU, Tsuji DH, Imamurra R, Sennes LU. Variação da intensidade vocal: estudo da vibração das pregas vocais em seres humanos com videoquimiografia. Rev Bras Otorrino. 2003;69(4):464-70. https://doi.org/10.1590/S003472992003000400005

13. Ferrand C. Relationship between masking levels and phonatory stability in normal-speaking women. J Voice. 2005;20(2):223-28.

14. Lauer MC. Comportamento vocal diante do efeito Lombard em mulheres com disfonia funcional. São Paulo. Monografia [Especialização em Voz] - Centro de Estudos da Voz; 2006.

15. Burnett TA, Senner JE, Larson CR. Voice F0 responses to pitch-shifted auditory feedback: a preliminary study. J Voice. 1997;11(2):202-11. https://doi.org/10.1016/j.jvoice.2005.04.004

16. Caldeira CRP, Vieira VP, Behlau M. Análise das modificações vocais de repórteres na situação de ruído. Rev Soc Bras Fonoaudiol. 2012;17(3):321-6. https://doi.org/10.1590/S151680342012000300014

17. Anjos ML. Incidência de disfonia no professor [Monografia]. Salvador: Centro de Especialização em Fonoaudiologia Clinica; 1999. 
18. Pereira PM, Ricz HMA, Aguiar-Ricz LN. Medidas acústicas e autopercepção de mulheres jovens em prova de fala continua. In: $19^{\circ}$ Seminário Internacional de Iniciação Científica e Tecnológica da USP; 2011 [Acesso em: 6 jul 2014]; Ribeirão Preto. Disponível em: https://uspdigital.usp.br/siicusp/cdOnlineTrabalhoVisualizarResum o? numeroInscricaoTrabalho $=1991 \&$ numeroEdicao $=19$

19. Burguetti FAR, Carvallo RMM. Sistema auditivo eferente: efeito no processamento auditivo. Rev Bras Otorrinolaringol. 2008;74(5):73745. https://doi.org/10.1590/S0034-72992008000500016

20. Marotta RMB, Quintero SM, Marone SAM. Avaliação do processamento auditivo por meio de reconhecimento de dissílabo em tarefa dicótica SSW em indivíduos com audição normal e ausência do reflexo acústico contralateral. Rev Bras Otorrinolaringol. 2002;68(2):254-61. https://doi.org/10.1590/S003472992002000200016

21. Fávero ML, Sanchez TG, Bento RF, Nascimento AF. Asymmetrical cochlear activity: A CNS influence? Arq Otorrinolaringol. 2005;9(4):300-4.

22. Khalfa S, Bougeard R, Morand N, Veuillet E, Isnard J, Guenot M et al. Evidence of peripheral auditory activity modulation by the auditory cortex in humans. Neuroscience. 2001;104(2):347-58. https://doi.org/10.1016/S0306-4522(01)00072-0

23. Khalfa S, Morlet T, Micheyl C, Morgon A. Evidence of peripheral hearing asymmetry in humans: clinical implications. Acta Otolaryngol. 1997;117(2):192-6.
24. Axelsson A, Ringdahl A. Tïnnitus: a study of its prevalence and characteristics. Br J Audiol.1989;23(1):53-62. https://doi. org/10.3109/03005368909077819

25. Pirilä T. Left-right asymmetry in the human response to experimental noise exposure. II. Pre-exposure hearing threshold and temporary threshold shift at $4 \mathrm{kHz}$ frequency. Acta Otolaryngol. 1991;111(4):861-6.

26. Khalfa S, Collet L. Functional asymmetry of medial olivocochlear system in humans: towards a peripheral auditory lateralization. Neuroreport. 1996;7(5):993-6. https://doi.org/10.1097/00001756199604100-00008

27. Ciuman PR. The efferent system or olivocochlear function bundle: fine regulator and protector of hearing perception. Int J Biomed Sci. 2010;6(4):276-88.

28. Lincoln M, Packman A, Onslow M. Altered auditory feedback and the treatment of stuttering: a review. J Fluency Disord. 2006;31(2):71-89. https://doi.org/10.1016/j.jfludis.2006.04.001

29. Prado AC. Principais características da produção vocal do deficiente auditivo. Rev CEFAC. 2007;9(3):404-10. https://doi.org/10.1590/ S1516-18462007000300014

30. Guidini RF, Bertoncello F, Zanchetta S, Dragone MLS. Correlações entre o ruído ambiente em sala de aula e a voz do professor. Rev Soc Bras Fonoaudiol. 2012;17(4):398-404. https://doi.org/10.1590/ S1516-80342012000400006 